\title{
Effect of Forbush decreases on the latent atmosphere energy
}

\section{Timofeev V.E.}

Affiliation Yu. G.Shafer Institute of Cosmophysical Research and Aeronomy, SB RAS, Yakutsk, Lenin 31 ave., Russia

E-mail: vetimofeevlikfia.ysn.ru

\section{Timofeev L.V. ${ }^{1}$,}

Affiliation Yu. G.Shafer Institute of Cosmophysical Research and Aeronomy, SB RAS,

Yakutsk, Lenin 31 ave., Russia

E-mail: levtimofeevlikfia.ysn.ru

\section{Samsonov S.N.,}

Affiliation Yu. G.Shafer Institute of Cosmophysical Research and Aeronomy, SB RAS,

Yakutsk, Lenin 31 ave., Russia

E-mail: s samsonoveikfia.ysn.ru

It has been shown that cosmic rays may affect the aggregate transitions of water in the free atmosphere through ionization of air and, thereby, the atmospheric parameters. The pressure variations derived from measurements of the water content across the atmosphere have been estimated. According to these estimates, the magnitude of possible pressure variations caused by cosmic rays in the form of Forbush decreases of galactic cosmic rays is $\approx 4.8$ mbar. Data on the near-Earth pressure at three considerably distant points (Moscow, Apatity, and Yakutsk) have been analyzed in order to prove this effect. It follows from an analysis that the effect of galactic cosmic rays on the atmosphere is simultaneously observed at least in polar and middle latitude regions of the Earth.

Keywords: cosmic rays, atmospheric parameters, surface pressure

35th International Cosmic Ray Conference - ICRC2017

12-20 July, 2017

Bexco, Busan, Korea

\section{${ }^{1}$ Speaker}




\section{INTRODUCTION}

Water in the atmosphere (from the upper boundary up to its lower one) is represented in the form of three following components : a) separate molecules ; b) clusters and as a part of the compounds representing heteronuclear particles and microscopic aerosols (many of them can serve as nuclei of condensation and sublimation of water steam and also affect a transmission of radiant energy of the Sun); c) small droplets and crystals of ice which also essentially affect a transmission of radiant energy of the Sun.

Each of the components is supported by a certain chain of physical-chemical processes in a cut of the atmosphere. One of them is connected with ionization. For example, with decreasing ionization the processes of recombination of heavy ionic clusters will predominate, and their concentration will decrease. As a result, small droplets of water which have been formed on such clusters, will immediately evaporate ("will dry up"). Small uncharged droplets of water cannot exist in one atmosphere [Matveev, 1965; Skryabin, 1979]. Simultaneously the water clusters formed on ions, will break down into separate molecules of water that will decrease the efficiency of processes of condensation and sublimation. And it, in its turn, will lead to the decrease of efficiency of the formation processes of larger droplets and ice crystals capable to form parts of fog (cloud). All it happens in the reverse order if ionisation will increase .

As a result of such processes a fraction of water existing in the atmosphere in the form of separate molecules, will vary in a sufficiently wide limits that will result in changes of the ground pressure. (It increases when ionization decreases because the water fraction existing in the form of separate molecules will increase. It decreases when ionization increases because water molecules will be a part of clusters and, as a result, the molecules will form small droplets, for example, fog parts in the clouds).

All it leads to some changes of a traditional change of latent energy of the atmosphere because it is connected with a state of aggregation of water (small droplets or separate molecules). Galactic cosmic rays (GCRs) penetrate into the lower atmosphere. Under the change of their intensity some atmospheric parameters will vary. Considering the above mentioned, the problem is posed - to reveal the value and a type of a " response" in variations of the ground pressure (over the Apatity, Moscow and Yakutsk) on exposure to GCRs in the form of Forbush-decrease of their intensity.

\section{SELECTION, PROCESSING AND ANALYSIS OF THE OBSERVATIONAL SET OF DATA}

Principally, the GCRs intensity undergoes little changes but on their background there are considerable increases ( flares of solar cosmic rays) and Forbush- decreases of GCRs. The first ones are sufficiently rare events and they are mainly observed in polar areas. They cover smaller 
squares of the planet than Forbush- decreases. Besides that, their duration is very small (minutes, hours). For the appreciable effect from the release of latent energy on major squares of the planet the times of some days are required. Therefore, to study the effect of cosmic rays on the ground pressure at the latitudes remote from auroral zone but at which Forbush-effects are noticeably manifested, two points, Moscow and Yakutsk, were chosen. They are removed in longitude but they are within middle latitudes. The point of Apatity which is close to the auroral zone has been taken for comparison .

The effects of Forbush-decreases can be repeated in 27 days in the slackened and distorted form. If there is a connection between changes of the cosmic ray intensity and ground pressure then such repetitions can bring considerable distortions into the results of experimental data processings. Besides, we expect that changes in the ground pressure because of cosmic rays will be more prolong by several fold than because of auroral precipitations (the effect because of auroral precipitations usually does not exceed 2-3 days). The duration of "response" in the ground pressure because of cosmic rays is expected in the interval of $\approx 5-27$ days.

Fig. 1 presents the temporal change of the ground pressure at Moscow from January 1, 1988 to December 31, 1997. The first day is taken as a unit.

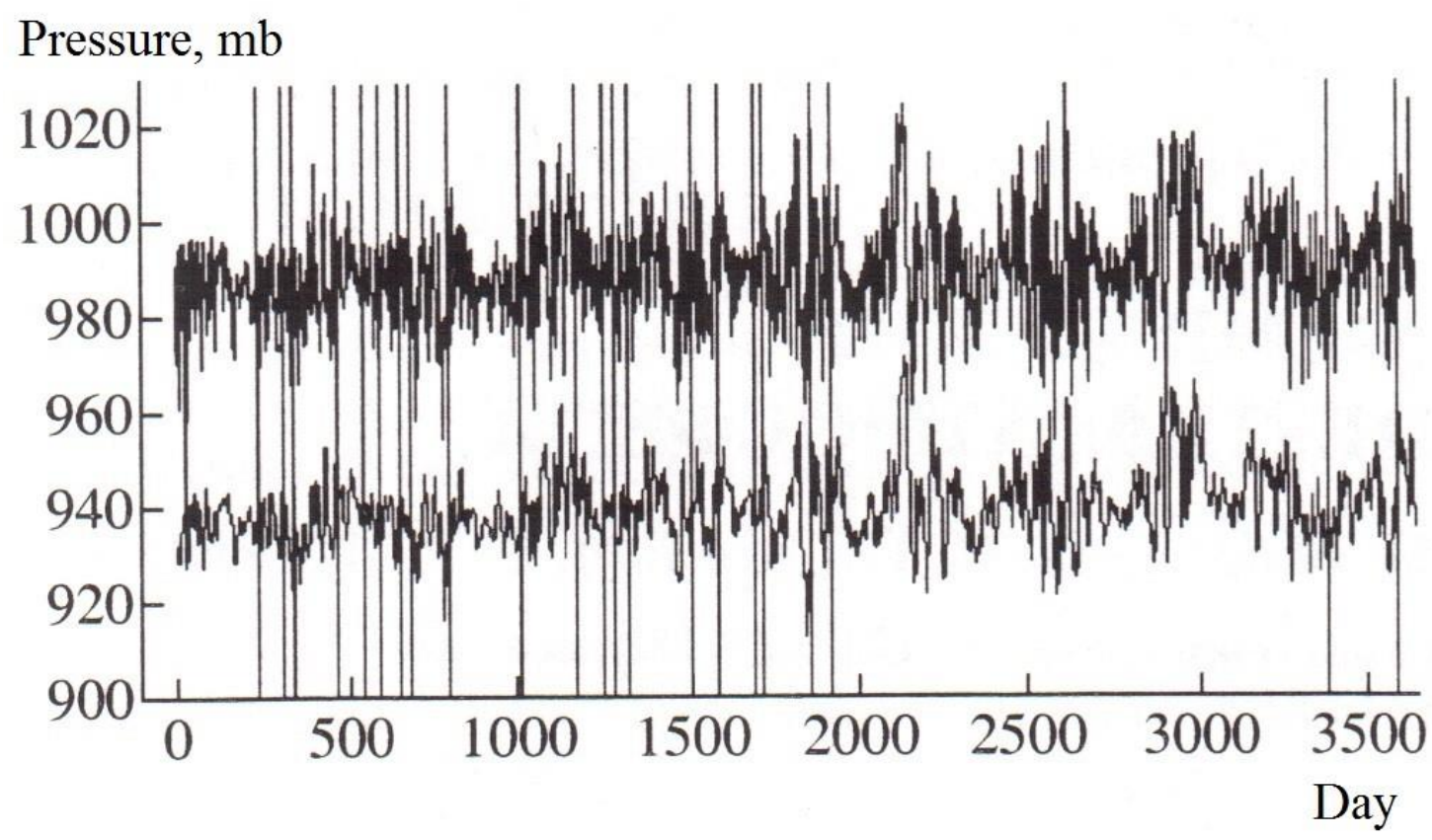

Fig. 1. Temporal change of the ground pressure at Moscow from January 1, 1988 to December 31, 1997. The lower plot presents the 5-day trend from the data of the upper plot shifted by $50 \mathrm{mb}$. The onsets of the basic phase of Forbush-decrease are marked by thin verticals.

As is seen from Fig. 1 (the upper plot) that the greatest fluctuations of ground pressure at Moscow are in the interval up to $\approx 5$ days and also in the seasonal change. If we suppose that the expected amplitude of effect is $\approx 1 \mathrm{mb}$ then, as is seen from this plot, the fluctuations with periods less than 5 days are more by a factor of approximately ten than the expected effect, and the seasonal change is more by a factor of approximately twenty than the expected effect, nevertheless it is noticeably manifested almost in every, separately taken case. It is seen on the lower plot of the Fig. 1 which represents itself a 5-day trend from the upper plot data shifted by 
$-50 \mathrm{Mb}$ (the shift has been made in order to present both plots in one Figure ). Thin verticals in Fig. 1 correspond to the time moments of the onset of Forbush- decreases by the data of the Yakutsk neutron monitor. Almost after each such line in the range of 5-27 days the increase of pressure in the form of burst of $\approx 10$ day duration takes place. For graphic example some part of the scale in Fig. 1 is presented on the expended scale in Fig. 2.

As is seen from Fig. 2 such burst of pressure is manifested almost after each onset of the main phase of Forbush- decrease. In this case, it is manifested both on "increase" of the temporal change of pressures (see Fig.2), (for example, in the vicinity of the first line) and on the "decrease" ( the second, the third and forth lines). It is also noticeable " inside" intervals of general decrease of pressure (for example, in a vicinity of the sixth line). In attempting to find an average value, for example, from a sum of changes of pressure near the third and fourth lines, very major errors exceeding the expected effect by a factor of several times appear. For their weakening we take a derivative from the lower plot of Fig.1 and using a superposed epoch technique for the period of 10 days before and 27 days after we will find a temporal change of average derivative (the moments of onset of Forbush-decreases are marked by verticals). At such processing the influence of average values near such lines will be equal to zero because the derivatives from constants are equal to zero. Besides, the influence of general increase - decrease will be considerably slackened. It is caused by the fact that the number of cases of increase decrease is approximately equal. Further, the value of derivatives describing the effect, is several times more than the derivatives describing the systematic increases -decreases. The obtained average derivative should be integrated. As a result we will obtain changes, on the average, for the period before 10 days and 27 days after the onset of Forbush-decrease in which the influences of mean values will be absent, and the influences of systematic increases -decreases in the average intervals will be slackened

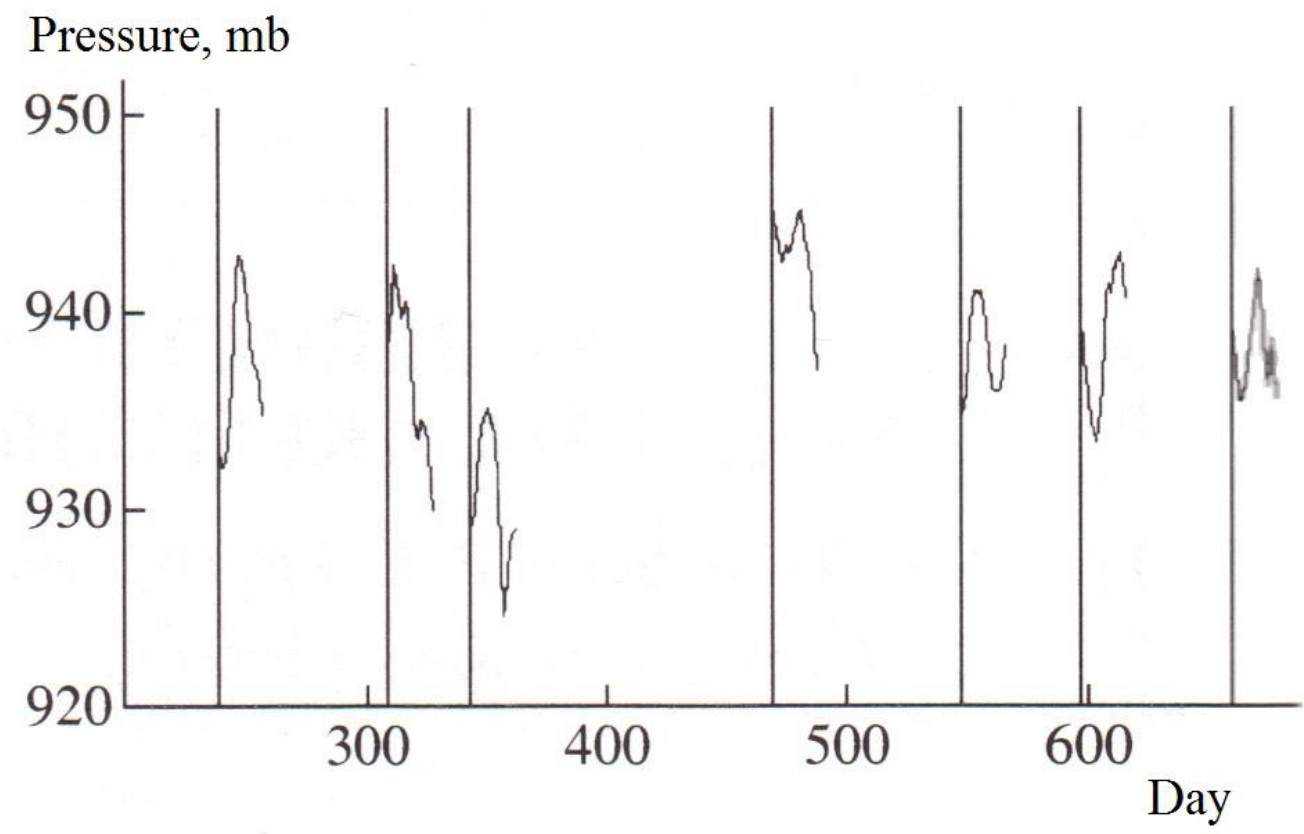

Fig. 2. Temporal change of the ground pressure near to the moments of onset of the main phase of Forbush-decrease (only a part of the scale is shown).

For Moscow such changes are shown in Fig. 3 (the third plot from above). The similar 
technique has been used a set of data for. Yakutsk (the lower plot) and Apatity (the second plot from above). The upper plot of Fig. 3 presents the average Forbush-effect normalized to the 5700 account and obtained by using a superposed epoch technique near the reference lines.

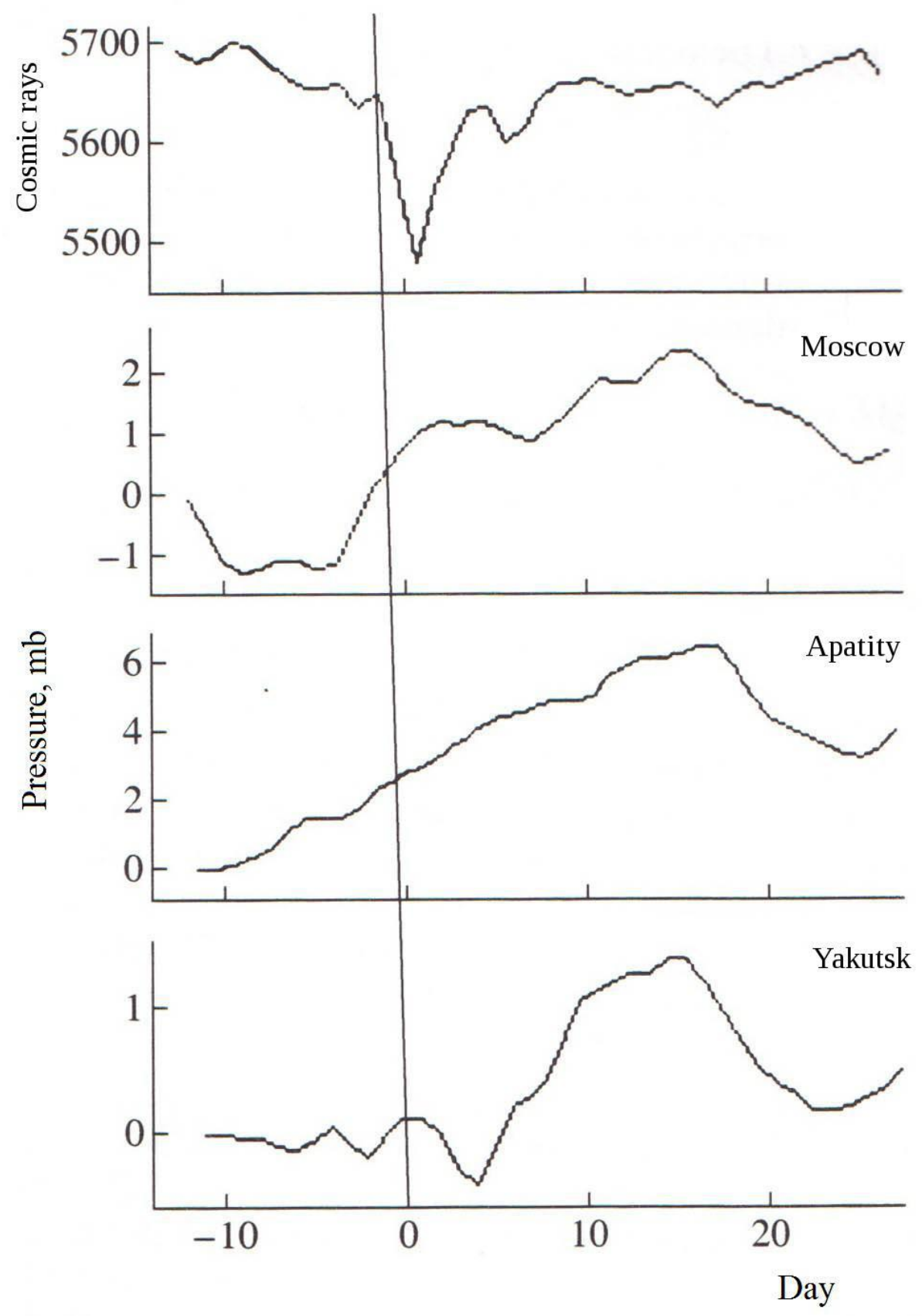

Fig. 3. Temporal change of the cosmic rays and ground pressure at Apatity, Moscow and Yakutsk.

As is seen from Fig. 3, the value of changes of the ground pressure at various longitudes and latitudes is different : the least value is at Yakutsk $(\approx+1.5 \mathrm{mb})$, the greatest one is at Moscow $(\approx+6 \mathrm{mb})$. Close to the auroral zone at longitudes of eastern Europe the effect is $\approx+3 \mathrm{mb}$. Despite 
so major distinctions of the value of effect, the general feature of temporal change and the position of its maximum remain almost invariable. The maximum is $\approx 13-14$ days "behind" the Forbusheffect .The temporal change of the pressure begins $\approx 3-5$ days earlier from the onset of main phase. It is , apparently, caused by the fact that, on the average, the temporal change of changes of cosmic rays begins earlier ( $\approx 7-10$ days before), than the onset of main phase of the Forbush-effect ( see Fig. 3, the upper plot). Thus, as the experiment shows, after the Forbush-decrease on the $\approx 13-14$ days there is the ground pressure increase of $\approx 1.5-6 \mathrm{Mb}$ almost on the whole square of Russia. Probably, the effect is propagated to the middle and polar latitudes over the whole northern and southern hemispheres of the Earth.

It is necessary to show that the considered effect, even if in principle, is possible. This problem is sufficiently complicated . Really, it is necessary to investigate two possibilities. The first one should show that, in principle, the conditions for generation of effective condensation nuclei at the expense of iof the substance ionization of the atmosphere by GCRs. The second one should show that under the existing physical-chemical conditions in the atmosphere the effect of change of the pressure can reach the value of the order of millibar units. Below we will try to investigate both possibilities.

\section{RELEASE OF THE LATENT ENERGY AT THE EXPENSE OF IONIZATION OF THE SUBSTANCE OF THE COSMIC RAY ATMOSPHERE BY COSMIC RAYS}

The substance of air in the troposphere is mainly ionized by GCRs. Therefore, one should expect that the noticeable proportion (and, probably, all their quantity) of such aerosols is formed by cosmic rays. In brief, the principle of their formation is the following. Cosmic rays in the ionization of neutral components of the atmosphere and in the ion-molecular cycles ( the total number of such processes is more than 22 [Sochnev, 1977; Janes, Ress, 1973]) form the $\mathrm{O}_{2}{ }^{+}$and $\mathrm{NO}^{+}$ions. Particles $\mathrm{O}_{2}{ }^{+}$and $\mathrm{NO}^{+}$, in turn, initiate reactions with the formation of heavy ions (the total number of such reaction is $\approx 12$ [Ferguson, 1972; Ferguson and Fehsenfeld, 1979]). One of the products of such reactions are clusters of hydronium $\mathrm{H}_{3} \mathrm{O}^{+}\left(\mathrm{H}_{2} \mathrm{O}\right)$ and $\mathrm{H}_{3} \mathrm{O}^{+}\left(\mathrm{H}_{2} \mathrm{O}\right)_{2}$.

The cluster of hydronium is very hygroscopic. Besides that, according to [Biondi, 1969] it has the lowest energetic threshold for the connection of water molecules . Therefore, they will be enlarged in accordance to the reaction [Bondi, 1969]:

$$
\mathrm{I}_{3} \mathrm{O}^{+}\left(\mathrm{H}_{2} \mathrm{O}\right)_{\mathrm{n}}+\mathrm{H}_{2} \mathrm{O}+\mathrm{M} \rightarrow \mathrm{H}_{3} \mathrm{O}+\left(\mathrm{H}_{2} \mathrm{O}\right)_{\mathrm{n}+1}+\mathrm{M}
$$

The heavy cluster of hydronium $\mathrm{H}_{3} \mathrm{O}{ }^{+}\left(\mathrm{H}_{2} \mathrm{O}\right)$ n possesses a catalytic properties for the start-up of fast redirecting reactions, for example, such as [Castleman, 1973]:

$$
\begin{aligned}
& \grave{I}_{3} \mathrm{O}^{+}\left(\mathrm{H}_{2} \mathrm{O}\right)_{n}+\mathrm{NH}_{3}+\mathrm{M} \rightarrow \mathrm{NH}_{4}{ }^{+}\left(\mathrm{H}_{2} \mathrm{O}\right)_{\mathrm{n}-1}+\mathrm{M}, \\
& \mathrm{NH}_{4}{ }^{+}\left(\mathrm{H}_{2} \mathrm{O}\right)_{\mathrm{m}}+\mathrm{HNO}_{3} \rightarrow \mathrm{NH}_{4} \mathrm{NO}_{3}\left(\mathrm{H}_{2} \mathrm{O}\right)_{n}+\mathrm{H}_{3} \mathrm{O}^{+}\left(\mathrm{H}_{2} \mathrm{O}\right)_{\mathrm{m}-\mathrm{n} .} \text { (2) }
\end{aligned}
$$


In the reactions (2) hygroscopic small parts $\mathrm{NH}_{4}+\left(\mathrm{H}_{2} \mathrm{O}\right) \mathrm{n}, \mathrm{NH}_{4} \mathrm{NO}_{3}\left(\mathrm{H}_{2} \mathrm{O}\right)$ n and new portions of clusters of hydronium $\mathrm{H}_{3} \mathrm{O}^{+}\left(\mathrm{H}_{2} \mathrm{O}\right)$ n are formed. They, in their turn, after restoration of the former value in the reaction (1) start catalytic processes up again (2). As if the chain reaction results which promptly increases the concentration of water steam condensation nuclei, namely, the small parts of $\mathrm{NH}_{4}{ }^{+}\left(\mathrm{H}_{2} \mathrm{O}\right)_{n}, \mathrm{NH}_{4} \mathrm{NO}_{3}\left(\mathrm{H}_{2} \mathrm{O}\right)_{n}$ and $\mathrm{H}_{3} \mathrm{O}{ }^{+}\left(\mathrm{H}_{2} \mathrm{O}\right)$.

As is seen from $(1,2)$, an " intensifying effect" of increase of concentration of clusters of the nitrate of ammonium and hydronium takes place. The amplification coefficient is equal to the number of repetitions of the reactions (2) which is determined by the number of molecules of $\mathrm{NH}_{3} \mathrm{HNO}_{3}$. According to [Karol, 1974; Hedy, 1970] the number of small particles of $\mathrm{NH}_{3}$ and $\mathrm{HNO}_{3}$ in the lower atmosphere is $10^{-8}-10^{-7}$ from the number of all air molecules . It is necessary to note that at such small fraction their number is huge. For example, in the upper layers of troposphere where, mainly, the absorption of cosmic rays (at heights of $\approx 6-8 \mathrm{~km}$ ) occurs, it is equal to $\approx 10^{11-} 10^{12}$ particles $/ \mathrm{sm}^{3}$.

And what concentration of the condensation nuclei of water steam should affect the release of the latent energy noticeably? Let's estimate it by the following reasons. The concentration of small parts of fog in the upper edge of clouds is of the order of $10 \mathrm{parts} / \mathrm{sm}^{3}$ [Matveev, 1965]. This value is less by a factor of 10-100 billions than the number of little parts of $\mathrm{NH}_{3}$ and $\mathrm{HNO}_{3}$ which support processes (2). Therefore, even if the efficiency of processes of the generation of aerosols by cosmic rays will be only $10^{-10}-10^{-11}$ then and in this case the number of condensation nuclei will make a value comparable with their background content. The suggested catalytic cycle (1-2) is not the only. Other cycles, for example, with participation of the components of "sea dust" [Castleman, 1973], sulfur-containing components etc. are also possible. The formation of aerosols under the influence of the ionizing fluxes, at least, for the upper atmosphere of planets, is an usual astrophysical phenomenon [Skryabin, 1979; Dauviller, 1959]. So, when increasing the solar activity which is accompanied by chromospheric bursts initiating strong aurorae on the Earth the reflected luminosity of the Venus increases. This effect is sufficiently strong and propagates even to the remote planets having the powerful atmosphere. For example, during the days of solar activity maximum the brightness of the Jupiter increases by approximately $20 \%$ [Dauviller, 1959]. All it indicates that cosmic rays through the formation processes of hygroscopic aerosols can affect the ground pressure.

But, unfortunately, observationally such connections on a region taken separately are difficult for revealing. Cosmic rays affect the ability of the atmosphere to reveal the latent energy. The effect is approximately equal and coincident on the whole square of planet. (At least, from middle latitudes to the poles). And this property makes difficult a search of their influence on the weather reorganisation. 


\section{ESTIMATION OF THE POSSIBLE VALUE OF EFFECT OF THE CHANGE OF PRESSURE UNDER THE INFLUENCE OF COSMIC RAYS}

Thus, further the problem reduces to the determination of effect of the change of pressure at the expense of transition of water from small droplets and charged clusters into water in the form of separate molecules. Such transition is included into a very compound circuit of physicalchemical and dynamic processes in the atmosphere. The complexity is caused by the fact that in this case in the atmosphere a huge energy ( $\approx 800-900 \mathrm{cal} / \mathrm{g}$ [Sedunov, etc. 1997]) is released or used which is transformed into dynamic processes.

Consider the elementary problem - an isothermal transition of substance of water in the homogeneous atmosphere from small droplets to molecules without transformation into dynamic processes. The pressure in the atmosphere is represented by a sum of partial pressures of various gases and microscopic aerosols $(P)$. For our problem we will present it in the form of sum of the pressure of dry air $\left(P_{c}\right)$, pressure of the water existing in the form of molecules $\left(\mathrm{P}_{\mathrm{M}}\right)$, and the pressure formed by microscopic aerosols $\left(P_{a}\right)$. (Hereinafter the case in point is aerosols which participate in the Brownian motion. Larger aerosols will simply fall out in a form of sediments).

Let's consider two extreme cases. When water in the atmosphere exists in the form of separate molecules then the atmospheric pressure will be:

$P_{l}=P_{c}+P_{m}$

If water exists in the form of microscopic aerosols then it is:

$P_{2}=R s+R a(4)$

Taking into account a Boltzmann constant these relations will become:

$P_{1}=R s+p m k T, P_{2}=P_{c}+n_{a} k T$,

where $n_{m}$ and $\mathrm{n}_{\mathrm{a}}$ is a concentration of molecules of water and the water aerosols existing in the free atmosphere.

The expected peak effect of the change of pressure will be :

$\Delta P=P_{1}-P_{2}=($ pm-pas $) k T$. (6)

As $n_{M} » n_{a}$ then it is reduced to the value :

$\Delta P=n_{m} k T$. (7)

To estimate $\Delta P$ we will use the annual average values of the content of water at heights of 5 and $9 \mathrm{~km}$ at the latitude $50^{\circ}$. They are equal to $6.14^{*} 10^{-6}$ and $10^{-6} \mathrm{~g} / \mathrm{cm}^{3}$ that it corresponds to concentrations of the molecules of water $2.04 * 10^{17}$ and $3.34 * 10^{16} 1 / \mathrm{cm}^{3}$, respectively. From these data of the content of water, at its transformation into separate molecules at the temperature $T=250-300^{\circ} \mathrm{K}$ there is the additional pressure at a height of $5 \mathrm{~km}(7.04-8.45) \mathrm{Mb}$, at a height of $9 \mathrm{~km}$ (1.15-1.38) Mb. In the range of 5-9 km approximately $50 \%$ of GCRs are absorbed. In the same interval the aggregate transition from the microscopic droplets of water to molecules 
can change the pressure, on the average, by $\approx 4.8 \mathrm{Mb}$. (The estimation is obtained from the extreme values of additional pressure at heights of 5 and $9 \mathrm{~km}$ ). It will be a value of expected effect. Dynamic processes can increase or decrease this value. And really, if the additional portions of moist air will arrive (or leave) into the field of heights of the effective absorption of space radiation then it will lead to the change of expected values. In this case the real effect can be more or less than the average expected effect by a factor of two-three.

\section{CONCLUSIONS}

The effect of influence of GCRs on the atmosphere covers, at least, polar and mid-latitude areas of the Earth. Thus, for example, Forbush-decreases cause the increase of pressure of about 1-6 mb. In this case the value of effect over various areas can differ by a factor of two-three (at Moscow is about $6 \mathrm{mb}$, at Yakutsk is $1.5 \mathrm{mb}$, at Apatity is $3 \mathrm{mb}$ ). At so major distinction in amplitude the form of temporal change of pressure has no considerable distinctions. In all three points the effect maximum falls at the 13-14 days from the onset of main phase of the Forbusheffect. Its mean duration at all points is approximately equal to 10-15 days. The ionization of air at the expense of GCRs can considerably affect the processes in which a generation of water aerosols takes place. In the atmosphere there are always the physical-chemical conditions under which, in the case of realization of aggregate transitions of water, there will be changes of approximately (1-6) $\mathrm{mb}$ in the ground pressure. In this case in the damper and warmer atmosphere such changes can be two-three times stronger.

The work was financially supported by the Russian Fund for Basic Research (the project № 01-02-17278, the project № 01-02-96206, the project №15-45-05090).

\section{REFERENCES}

1. Biondi M. A II Canad. J. Chemistry, 47. 1711 p. 1969.

2. Castleman A.W. Aerosol chemistry physics and chemistry of upper atmosphere//Ed. B.M. McCornac. Dordrecht-Holland. P. 143-157. 1973.

3. Dauviller A. Activite auroral et Albedo Planetares//Comptes Rendus Hebdomadaires des Seances de I'academie des Sciences V. 248. № 12. P. 1740-1743. 1959.

4. Ferguson E.E. Review of laboratory measurements of aeronomic ion-neutral reactions//Ann. Geophys V. 28. P. 389-395. 1972.

5. Ferguson E.E., Fehsenfeld F.C. Water vapour ion cluster concentrations in the dRegion//J. Geophys. Res V. 74. №24. P. 5743-5751. 1979.

6. Hedy T.V., McKay H.A.C., Pilbeam A., Seargill D. Ammonia and ammonium sulphate 
in troposphere over the United Kingdom//J. Geophys. Res V. 75. P. 2317-2322. 1970.

7. Janes RA., Ress M.N. Time dependent studies of the aurora. 1. Ion density and composition//Planet. Space Sci V. 21. № 4. P. 537-557. 1973.

8. Karol I.L. High-altitude planes and the stratosphere. - Leningrad: Gidrometeoizdat, 1974. - P. 48 .(In Russian).

9. Matveyev L.T. The fundamentals of general meteorology. Physics of atmosphere. Leningrad:Gidrometeoizdat, 1965. - P. 876 . (In Russian).

10. Sedunov Yu.S., Volnovitsky O.A., Petrov N.N., Rejtenbakh R. G, Smirnov V. I, Tchernikov A.A. The atmosphere. The reference book (reference data, models). Leningrad: Gidrometeoizdat , 1997. -509 p.(In Russian).

11. Skryabin N.G., Strod S.M., Moiseyev V.G. Possible mechanism of connection of some optical effects in the upper atmosphere with corpuscular intrusions// Study of optical radiation of the night sky / Ed. Nadubovich Yu.A. -Yakutsk: Yakutsk Detachment of Siberian Branch of the USSR Academy of Sciences, 1979.-P. 86-94. (In Russian).

12. Sochnev V. G, Tulinov V. F, YakovlevS.G. Some aspects of the effect of corpuscular radiation on the Earth's atmosphere under quiet and disturbed conditions//Effects of solar activity in the lower atmosphere / Ed. Rakitova A.P. - Leningrad: Gidrometeoizdat, 1977. -P. 47-54. (In Russian). 